\title{
An Agile Methodology at Telecommunications Morpho
}

\author{
Dr. Tiago F. Alwes de Olivei \\ Assistant Professor \\ Lakehead University, Canada
}

\begin{abstract}
It is winding up clear, not minimum from the pages of this distribution, that lithe advancement strategies are being embraced or possibly considered by a developing number of programming improvement groups and associations. Regardless of whether you are as of now a functioning specialist coordinated advancement, or thinking about its appropriation on your venture, you will know about the business benefits that can be determined through quicker and increasingly powerful programming conveyance also the persuasive effect it can have on improvement groups[1]. On the other hand, possibly you work for a substantial association that presently cannot seem to make any genuine advances into dexterous improvement and are left considering how deftness could be made to chip away at a vast scale. In case you're in the last camp, or regardless of whether you are not effectively thinking about spry improvement in that capacity but rather are attempting to convey expansive and/or complex projects utilizing customary methodologies and wishing there was a superior way, at that point you are most likely where British Telecom (BT) wound up in 2004. [2] That was previously the landing in the organization of another CIO who methodically started supplanting the organization's long-standing cascadebased conveyance forms with one that encapsulated the key standards of nimble conveyance. This article shows a review of the methodology taken by BT, outlining how light-footed advancement standards can be connected effectively at the venture level. Obviously, the methodology taken by BT is not for the timid - it has incorporated a high level of hazard, and surely a great deal of torment. Presently very much into its second year be that as it may, even though the change is a long way from finish, it is as of now paying profits.
\end{abstract}

\section{Index Terms: Agile, Telecommunications, Project management, Big Design, British Telecom, CIO, DSDM}

[1] Introduction: BT utilizes about 8,000 IT experts in an assortment of jobs including venture and conveyance the executives, design, and structure, programming building, joining, and testing, operational help and administration the board. A lot of its inside focused advancement work has generally been directed through various business-focused conveyance tasks or projects, extending from very little, straightforward improvements to substantial scale and complex business arrangements, the last tending to be the standard. [3] The prevalent conveyance approach, surely for the bigger conveyance programs, was particularly cascade based. The utilization of deft improvement practice, outstandingly DSDM and Scrum, was constrained to few genuinely little, independent advancement groups. BT was in certainty one of the establishing individuals from the DSDM [4]Consortium and took a functioning part in forming the strategy in its initial days. Regardless of effectively conveying various huge, complex arrangements into a dynamic, focused yet exceptionally controlled business condition, numerous critical change programs were attempting to convey any prominent outcomes in a worthy time span. As a feature of a CMMI-roused[5] enhancement system, endeavors had been made to formalize recognized best practice forms into a standard conveyance strategy. In 2004, this standard philosophy was being taken off when the new CIO clarified that a completely new light-footed methodology was required.

I. Downsides of the cascade: Support of momentum cascade-based practices was not by any stretch of the imagination the appropriate response in any case. A significant number of the conveyance issues experienced at BT, [6] and no uncertainty other expansive associations, originate from the idea of the cascade lifecycle itself. A few instances of these issues are given here. For an increasingly entire pulverization of cascade rehearses, allude to Craig Larman's phenomenal work.

II. Poor necessities to catch : Catching necessities positively is certainly not an awful thing. On common extensive projects, be that as it may, [7]Singular business partners are on edge to join most of their known necessities into the first/next discharge "Gold clients" produce hundreds, if not a huge number of definite prerequisites that regularly bear little relationship to the business issues that should be tended to.

III. Most if not all prerequisites are given a high need: The prerequisites themselves, best case scenario, speak to the present view, which will absolutely have changed when the necessities are really executed

IV. Separated structure : Given the sheer number of necessities, the structure network ends up investing most if its energy endeavoring to make sense of what they mean. In the interim, the necessities investigators proceed onward to different tasks, taking with them critical implicit information. A few partners wind up worried that their prerequisites are not being sufficiently tended to, and in this manner decline to close the structures. Different partners uncover more prerequisites or raise change demands, occupying rare plan aptitude onto affect examinations

V. Advancement crush : With the plan organize having slipped, advancement groups wind up under extraordinary strain to convey segments into the incorporation condition by the initially concurred date. They regularly take the choice, reluctantly, [8]to begin advancement against an insecure plan, as opposed to do nothing or redirect assets to different projects. Framework testing is stopped with the goal that unique timescales are met, and the program apparently is on target.

VI. The coordination cerebral pain: The coordination group has a set number of weeks amid which it needs to incorporate what it hopes to be completely useful and generally sans bug code. Considering the unsteadiness of the part code, and the absence of any compelling relapse test capacity, exertion is rather redirected to attempting to determine rudimentary bugs in the conveyed code, liaising with an improvement group that is presently occupied with the following real discharge. [9]Real combination thusly 
keeps running into months, making a thump on impact on different projects requiring the administrations of the Integration group, also dissatisfactions inside the business network who had been caught up with setting themselves up for an on-time conveyance.

VII. The arrangement bad dream: It is currently no less than 6 , or even 12 - year and a half since the business initially recognized the requirement for this specific arrangement. Bargains and oversights made amid the prerequisites and configuration stages, trailed by de-perusing amid improvement has brought about an answer that bears little association with what was initially imagined. Additionally, the world has really proceeded onward meanwhile. The business at that point finds that the arrangement is not fit-for-reason and declines to embrace it. More awful, they receive it and before long find that it is moderate, blunder inclined and needs key highlights, and inevitably return to the old framework. [10]The final product - more shelfware! Right off the bat in every conveyance cycle, the program sets out clear focuses for what it hopes to accomplish for the business amid that cycle. These objectives perpetually incorporate a solid accentuation on the end-client encounter, for example, reaction times, exchange achievement rates, etc. Toward the finish of the cycle, the program is surveyed against these objectives, and the result of this appraisal will impact the planning of extra installments for the program colleagues. Projects neglecting to convey business esteem over a progression of cycles confront being shut down by and large. This obviously puts a specific measure of weight on the (interior) client to be clear about the business needs and the highlights that would give the best rate of profitability. It likewise necessitates that the client is prepared and ready to send the arrangements into the business and understand the planned advantages. By and by, programs frequently take at least two 90-day cycles to advance a specific answer for a point where it is fit for arrangement. All things considered, there is an open door toward the finish of each cycle to survey what has been conveyed up until this point, and to give input dependent on what has just been produced.

[2] Early Reflections: Despite some chaos in the starting \& some aching disappointments amongst some of the prior hot households and the delivery cycles, the new performs have now developed putative as the norm through British Telecom. Now well into the second year of its move from a waterfall system to the agile delivery performs, few of the public would be prepared to return to pre-Agile applies. In fact, most plans are now in search of ways of sanitizing their distribution procedures further by accepting truly iterative and a test-driven expansion methods within each delivery cycle. Though, some annotations would be worth noticing. Whenever you are boarding on an agile system delivery approach at the initiative level, it is imperious to speedily launch a 'critical mass' of persons who not only clutch the concepts behind it but are also contented with its submission. To begin that critical mass, you will possibly need to fit to help from the outside. So many consultancies are now specializing in the implementation of agile system performs within the large administrations. [11] British Telecom chooses to use two different corporations, each of which carried different strengths and viewpoints. Further to this, it is also indispensable establishing a sturdy central team to deliver ad-hoc provision, cherish the new methods, and to actively backing the new performs. Assured agile system practices like test-driven expansion are very much stiffer to adopt once most of your expansion is based on legacy code and outwardly sourced mechanisms. Continuous incorporation develops tremendously multifaceted when some of your main mechanisms are united across multiple databases. Some of British Telecom's programs are now following test-first and unremitting integration methods but as this takes time \& speculation and is only being done on a discriminatory basis. For Agile Development methods to work at the innovativeness level, you still require to pay due consideration to your systems construction. "Big Design Up-Front" (BDUF) cannot request to the agile pedant, but re-factoring of an enterprise construction simply is not applied. Not all transport activity fits precisely into the agile system expansion model. Given a choice, the normal inclination is to follow most actions using the traditional methods where you can continuously find some reason why "the new approach" is not suitable on your development. If you to this path, agile distribution will at best become a niche movement. [13] At British Telecom, a strong command confirmed that all packages put the new performs to the test whether this appeared reasonable or illogical. This facilitated to break through "pain barrier" and to guarantee that the new performs were given a real chance of attractive grip.

To be actually operative, the agile system method needs to grasp right across the full trade and not just the IT association. You might assume that the trade would be motivated at the view of having regular distributions of appreciated functionality. Though, a business also requests to move away from traditional waterfall model system performs \& change how it involves with the IT association. It must place its faith in the IT [14] organization that it will be delivered as guaranteed. It then requires to authorize that it is geared up to feat the distributions to gain extreme business profit. Applying the ideologies pronounced here on large schemes or packages in typical large administrations needs courage, fortitude, and no small degree of danger. Such a radical policy necessitates complete assurance from very top.

[3] Conclusion: Reorienting process of a large IT association from following well recognized waterfall-based method to be actually agile distribution unit takings endurance and time as well as a lot of promises. In British Telecom where the opening steps towards creativity agile delivery based were taken in late 2004, there has been an obvious and conclusive shift away from waterfall model system thinking. It has also transmuted quite drastically, the traditional utility of the IT division as a provider of IT facilities to the IT as essential to all major business enterprises. Above all it has formed an assertiveness, bordering on fascination, of delivering real value to the business through IT. Although the early achievements received, it is clear within British Telecom that there is still a very much long way to go before it can deliberate itself to be truly responsive. For any large association, the journey from waterfall system to agile system can be very long and stimulating. As with other supporters of Agile Expansion however, few at British Telecom would want to turn back to the old days again. 


\section{References:}

[1] DaYong Sang, Wang Ying and LiHua Wu, "Agile Software Development Methods and Practices" in , Xi-an:XIDIAN University press, pp. 160-180, 2010.

[2] Kurt Bittner, JingWen Luo, CanFeng Luo and HongYi Zhang, "Managing Iterative Software Development Projects" in , Beijing: Tsinghai university press.2010, pp. 109-130.

[3] PengZhiNan, "Agile development in software development application research", Chengdu: university of electronic science and technology, 2009.

[4] Shen Lei and Bei Jun Shen, "Research and Practice of Agile methods", Computer engineering, vol. 30, no. 7, pp. 219, 2005.

[5] XiaoHua Wang, "Software Reliability analysis and relevant problems study in agile development environment" in , Guiyang:GUI Zhou University, 2008.

[6] XiangYang Wang, "Limit research and application" in, Wuhan:Wuhan University, 2004.

[7] Cohn Mike and Song Rui, "Agile estimation and planning" in , Beijing:Tsinghai university press, pp. 36-87, 2007.

[8] Mo Ying, "Duty gives birth to the Dao-trace the source of agile development", Journal of programmers, no. 8, pp. 52, 2007.

[9] Xiao Qing Zhang and Hu Wei, "Shallow Judicators about Instant Acceptance in Agile Development Application", Programmer, vol. 3, 2009.

[10] XiaoYi Wang, "Reconstruction Secret of Agile Developing", Software World, pp. 21-59, 2007.

[11] Yang Na and YiHui Zhou, "The Agile Development and Reconstruction", Fujian Computer, pp. 12-138, 2008.

[12] C.M Robert, Martin, Micah Martin. Deng Hui and Sun Ming, "Principle Mode and Practice of Agile Software Development" in , Posts \& Telecom Press, pp. 123-156, 2008.

[13] DaYong Sang, "Requirement Analysis of Agile Development Process", Programmer, vol. 2, pp. 70-75, 2009.

[14] Negotiating Structural and Technological Change · Volume 23 By Brian Bolton, International Labour Office · 1993 